\title{
National versions of fibromyalgia questionnaire: Translated protocols or validated instruments?
}

\author{
Seppo Soinila \\ Department of Neurology, Turku University Central Hospital, Turku, Finland
}

Fibromyalgia remains a considerable challenge for both clinicians and researchers. In spite of established criteria [1], diagnostic accuracy of fibromyalgia seems to be far from satisfactory, there being both overdiagnosing and neglect of obvious cases. Perhaps even more significant, the assessment of fibromyalgia patients for the impact of the disease on physical and mental function, working capability, or quality of life includes large case-to-case variability. This may lead to biased judgement by treating physicians, consultants and decision-making authorities. Since the benefits offered by social security system are based on impaired function rather than the diagnosis itself, unequivocal and multi-dimensional description of the impact of fibromyalgia is crucial. Although careful informal description of the patient's status may include sufficient information, the ideal method would be based on standardized, validated assessment paradigm.

Pain is the major symptom of fibromyalgia. According to an early study of pain descriptors, the vocabulary of English-speaking pain patients contains 124 or 145 words for characterizing pain, depending whether the pain is associated with somatic or psychiatric etiology, respectively [2]. One of the first examples of a translated pain questionnaire stems from conversion of the classic McGill Pain Questionnaire [3] into Finnish. The Finnish equivalents of pain words were first defined by dictionary, the list was complemented by 76 persons and the final word list was ordered according to mean VAS value given on each word by each evaluator [4]. This demonstrates how intimately any pain-related assessment is bound to language. Beside pain, many other parameters of fibromyalgia examined by questionnaires depend on language and culture, making it necessary to validate translated questionnaires in the local context.

Fibromyalgia Impact Questionnaire (FIQ) [5] has been available for 20 years and has been translated into several languages except for Finnish. Gauffin and collaborators [6] now fulfill this gap by validating the Finnish version, Finn-FIQ. Their study includes 162 fibromyalgia patients obtained from a single hospital with long tradition on fibromyalgia research and with remarkably large recruitment population of 270,000 inhabitants. The material represents purest possible M79.0 cases, since confounding causes of

DOI of refers to article: 10.1016/j.sjpain.2011.10.004.

E-mail address: Seppo.Soinila@tyks.fi pain and disability have been carefully excluded. This is one of the largest materials among validation studies of FIQ published in any language.

All items were linked to the International Classification of Functioning, Disability and Health. The results were compared with six pain-related assessment protocols available in Finnish, two of them validated previously in Finnish, plus the patients own estimate of their general well-being. Good correlation was observed between the Finn-FIQ and the Rand-36 item health survey, the Health Assessment Questionnaire or the patients' self-assessment of general well-being. Results of Finn-FIQ were stable when repeated after six months both for newly diagnosed cases and those of longer duration ( $>2$ years).

Undoubtedly, Finn-FIQ being now validated as a multidimensional assessment tool and showing exceptionally high response rate among patients, will find extensive use both among Finnish doctors treating fibromyalgia patients and those studying fibromyalgia in Finnish-speaking population. Added value of specific language-based validation for non-Finnish-speaking pain doctors may arise from the authors' critical reconsideration of the original version, in which they point out variance in the response rate of some sub-items. In addition, some fine-tuning of the original questionnaire was made, based on obvious gaps in the previous versions validated in other languages.

We are looking forward to further studies showing the sensitivity of Finn-FIQ to detect changes caused by treatment interventions.

\section{References}

[1] Wolfe F, Smythe HA, Yunus MB, Bennett RM, Bombardier C, Goldenberg DL, Tug well P, Campbell SM, Abeles M, Clark P, Fam AG, Farber SJ, Fiechtner JJ, Franklin CM, Gatter RA, Hamaty D, Lessard J, Lichtbroun AS, Masi AT, Mccain GA, Reynolds WJ, Romano TJ, Russell IJ, Sheon RP. The American College of Rheumatology 1990 criteria for the classification of fibromyalgia. Report of the multicenter criteria committee. Arthritis Rheum 1990;33:160-72.

[2] Agnew DC, Merskey H. Words of chronic pain. Pain 1976;2:73-81.

[3] Melzack R. The McGill Pain Questionnaire: major properties and scoring methods. Pain 1975;1:277-99.

[4] Ketovuori H, Pöntinen PJ. A pain vocabulary in Finnish - The Finnish Pain Questionnaire. Pain 1981;11:247-53.

[5] Burckhardt C, Clark S, Bennet R. The fibromyalgia impact questionnaire: development and validation. J Rheumatol 1991;18:728-33.

[6] Gauffin J, Hankama T, Kautiainen H, Arkela-Kautiainen M, Hannonen P, Haanpää M. Validation of a Finnish version of the Fibromyalgia Impact Questionnaire (Finn-FIQ). Scand J Pain 2012;3:15-20. 